Vasile Ene, Ovidius University Constanţa, Romania Current address: 23 August 8717, Jud. Constanţa, Romania

e-mail: ene@univ-ovidius.ro

\title{
RIESZ TYPE THEOREMS FOR GENERAL INTEGRALS
}

\begin{abstract}
The author gives a general descriptive definition for integration, denoted by $\mathcal{P}$, which has as special cases the Lebesgue integral for bounded measurable functions, the Lebesgue integral, the Denjoy-Perron integral $\mathcal{D}^{*}$, the wide Denjoy integral $\mathcal{D}$, the Foran integral, the Iseki integral and the $S \mathcal{F}$-integral ([5]). This $\mathcal{P}$-integral will admit Riesz type representation theorems (introducing an Alexiewicz norm, and identifying $f$ with $g$ whenever $f=g$ a.e. on $[a, b])$. The classical Riesz representation theorem for the linear and continuous functionals on $\left(C([a, b]),\|\cdot\|_{\infty}\right)$ is a consequence of Theorem 2 .

In addition it is shown that the space of $\mathcal{P}$-integrable functions is of the first category in itself (see Section 5). Also a characterization of the weak convergence on this space is given.
\end{abstract}

\section{Introduction}

Our purpose is to define a suitable general descriptive definition for integration, denoted by $\mathcal{P}$, which has as special cases the Lebesgue integral for bounded measurable functions, the Lebesgue integral, the Denjoy- Perron integral $\mathcal{D}^{*}$, the wide Denjoy integral $\mathcal{D}$, the Foran integral, the Iseki integral and the $S \mathcal{F}$ integral ([5]). This $\mathcal{P}$-integral will admit Riesz type representation theorems, i.e., introducing an Alexiewicz norm on the space of all $\mathcal{P}$-integrable functions, and identifying $f$ with $g$ whenever $f=g$ a.e. on $[a, b]$ (see Section 3 ) we obtain a characterization of the linear and continuous functionals on this space, see

Key Words: Alexiewicz norm, completion, essentially bounded variation, bounded slope variation, weak convergence

Mathematical Reviews subject classification: 26A39, 26A46, 26A45, 46A50, 54E52

Received by the editors August 21, 1996 
Lemma 4 and Theorem 2. The proof of Theorem 2 is based on Theorem 1 , and to prove Theorem 1 we use a technique of [12], p. 75. As a consequence of Theorem 2 it follows the classical Riesz representation theorem for the linear and continuous functionals on $\left(C([a, b]),\|\cdot\|_{\infty}\right)$.

Further, we also prove that the space of $\mathcal{P}$-integrable functions is of the first category in itself (see Section 5) and we give a characterization of the weak convergence on it.

\section{Essentially Bounded Variation and the Bounded Slope Variation}

Definition 1. ([14]). Let $P \subset[a, b]$ be a set of positive measure, and let $f: P \rightarrow \overline{\mathbb{R}}$ be a measurable function, finite a.e. .

- $f$ is said to be essentially upper bounded if there exists a real number $M$ such that the set $\{x \in P: f(x)>M\}$ has measure zero.

- $f$ is said to be essentially lower bounded if the function $-f$ is essentially upper bounded.

- $f$ is said to be essentially bounded if it is simultaneously essentially upper bounded and essentially lower bounded, i.e., there exists $M>0$ such that the set $\{x \in P:|f(x)|>M\}$ is of measure zero.

- Let $\sup _{e s s}(f ; P)=\inf \{M:\{x \in P: f(x)>M\}$ has measure zero $\}$ if $f$ is essentially upper bounded and $\sup _{\text {ess }}(f ; P)=+\infty$ if not. Define $\inf _{\text {ess }}(f ; P)$ similarly .

- Let $\mathcal{O}_{\text {ess }}(f ; P)=\sup _{\text {ess }}(f ; P)-\inf _{\text {ess }}(f ; P)$.

- Let $\mathcal{O}_{\text {ess }}(f ; X)=0$, whenever $X$ is a null subset of $P$.

- $f$ is said to be of essentially bounded variation (abbreviated $f \in E V B$ ) on $P$, if there exists $M>0$ such that $\sum_{i=1}^{n} \mathcal{O}_{e s s}\left(f ;\left[a_{i}, b_{i}\right] \cap P\right)<M$, whenever $\left[a_{i}, b_{i}\right], i=1,2, \ldots, n$ are nonoverlapping closed intervals with endpoints in $P$.

- Let $E V(f ; P)=\inf \{M: M$ is as above $\}$ if $f \in E V B$ on $P$ and let $E V(f ; P)=+\infty$ if not.

- Let $V(f ; P)=\inf \left\{M: \sum_{i=1}^{n}\left(f\left(b_{i}\right)-f\left(a_{i}\right)\right) /\left(b_{i}-a_{i}\right)<M\right.$, whenever $\left[a_{i}, b_{i}\right], i=1,2, \ldots, n$ are nonoverlapping closed intervals with endpoints in $P\}$ if $f \in V B$ on $P$ and let $V(f ; P)=+\infty$ otherwise. 
Lemma 1. Let $P$ be a dense subset of $[a, b]$ and let $f: P \rightarrow \mathbb{R}, f \in V B$. Then there exists $\tilde{f}:[a, b] \rightarrow \mathbb{R}$ such that $\tilde{f} \in V B$ on $[a, b], \tilde{f}_{\mid P}=f$ and $V(\tilde{f} ;[a, b])=V(f ; P)$.

Proof. Let $x \in[a, b) \backslash P$. Then $\lim _{y \searrow x, y \in P} f(y)$ exists and is finite (because $f$ is bounded on $P$ ). Suppose that the above limit does not exist; then there exists $\epsilon_{o}>0$ such that whenever $\delta>0$ there exist $x^{\prime}, x^{\prime \prime} \in(x, x+\delta) \cap P$ such that $\left|f\left(x^{\prime}\right)-f\left(x^{\prime \prime}\right)\right| \geq \epsilon_{o}$. For $\delta=1$ there exist $a_{1}, b_{1} \in(x, x+1) \cap P, a_{1}<b_{1}$ such that $\left|f\left(a_{1}\right)-f\left(b_{1}\right)\right| \geq \epsilon_{o}$. For $\delta=a_{1}-x$ there exist $a_{2}, b_{2} \in(x, x+\delta) \cap P$, $a_{2}<b_{2}$ such that $\left|f\left(a_{2}\right)-f\left(b_{2}\right)\right| \geq \epsilon_{o}$. Inductively we obtain a sequence $\left\{\left[a_{n}, b_{n}\right]\right\}, n=1,2, \ldots$, of nonoverlapping closed intervals with endpoints in $P$ such that $b_{1}>a_{1}>b_{2}>a_{2}>\cdots b_{n}>a_{n} \ldots$ and $\left|f\left(a_{n}\right)-f\left(b_{n}\right)\right| \geq \epsilon_{o}$. Therefore $\sum_{n=1}^{\infty}\left|f\left(a_{n}\right)-f\left(b_{n}\right)\right|=\infty$, which contradicts the fact that $f$ is $V B$ on $P$. Similarly we can prove that $\lim _{y} x_{x, y \in P} f(y)$ exists and is finite whenever $x \in(a, b]$. Let $\lim _{y \searrow x, y \in P} f(y)=f(x+)$ and $\lim _{y} x_{x, y \in P} f(y)=$ $f(x-)$. Define $\tilde{f}:[a, b] \rightarrow \mathbb{R}$ by

$$
\tilde{f}(x)= \begin{cases}f(x) & \text { if } x \in P \\ f(x+) & \text { if } x \in[a, b) \backslash P \\ f(b-) & \text { if } b \notin P\end{cases}
$$

Then $\tilde{f}_{\mid P}=f$ and $V(f ; P) \leq V(\tilde{f} ;[a, b])$. Suppose on the contrary that $V(f ; P)<V(\tilde{f} ;[a, b])$. Let $\epsilon>0$ be such that $\epsilon+V(f ; P)<V(\tilde{f} ;[a, b])$. Then there exists $a=t_{0}<t_{1}<\cdots<t_{n}=b$ such that $\sum_{i=1}^{n}\left|\tilde{f}\left(t_{i}\right)-\tilde{f}\left(t_{i-1}\right)\right|>$ $\epsilon+V(f ; P)$. We may suppose without loss of generality that each $t_{i}$ does not belong to $P$. Then for each $t_{i}$ with $i=0,1, \cdots n-1$ it follows that there exists $t_{i}^{\prime} \in\left(t_{i}, t_{i+1}\right) \cap P$ such that $\left|\tilde{f}\left(t_{i}\right)-\tilde{f}\left(t_{i}{ }^{\prime}\right)\right|<\epsilon /(4 n)$ and for $t_{n}$ there exists $t_{n}^{\prime} \in\left(t_{n-1}, t_{n}\right) \cap P$ such that $\left|\tilde{f}\left(t_{n}\right)-\tilde{f}\left(t_{n}{ }^{\prime}\right)\right|<\epsilon /(4 n$. Therefore $\epsilon+V(f ; P)<\sum_{i=1}^{n}\left|\tilde{f}\left(t_{i}\right)-\tilde{f}\left(t_{i-1}\right)\right|=\sum_{i=1}^{n} \mid \tilde{f}\left(t_{i}\right)-f\left(t_{i}{ }^{\prime}\right)+f\left(t_{i}{ }^{\prime}\right)-f\left(t_{i-1}{ }^{\prime}\right)+$ $f\left(t_{i-1}^{\prime}\right)-\tilde{f}\left(t_{i-1}\right)\left|<2 n \cdot \epsilon /(4 n)+\sum_{i=1}^{n}\right| f\left(t_{i}{ }^{\prime}\right)-f\left(t_{i-1}^{\prime} \mid<\epsilon / 2+V(f ; P)\right.$, a contradiction.

Lemma 2. Let $f:[a, b] \rightarrow \overline{\mathbb{R}}$ be a measurable function. The following assertions are equivalent:

(i) $f \in E V B$ on $[a, b]$,

(ii) There exists $\tilde{f}:[a, b] \rightarrow \mathbb{R}$, such that $\tilde{f} \in V B$ and $\tilde{f}=f$ a.e. on $[a, b]$. Moreover $E V(f ;[a, b]) \leq V(\tilde{f} ;[a, b]) \leq 2 \cdot E V(f ;[a, b])$.

Proof. $(i) \Rightarrow(i i)$ We may suppose that $[a, b]=[0,1]$. For $n \geq 2$ let 


$$
\begin{aligned}
& \pi_{n}^{\prime}=\left\{\left[\frac{i}{2^{n}}, \frac{i+1}{2^{n}}\right]\right\}, i=0,1,2, \ldots, 2^{n}-1 ; \\
& \pi_{n}^{\prime \prime}=\left\{\left[0, \frac{1}{2^{n}}\right],\left[\frac{2 i-1}{2^{n}}, \frac{2 i+1}{2^{n}}\right],\left[\frac{2^{n}-1}{2^{n}}, 1\right]\right\}, i=1,2, \ldots, 2^{n-1}-1 ; \\
& M_{n, i}^{\prime}=\sup _{\text {ess }}\left(f ;\left[\frac{i}{2^{n}}, \frac{i+1}{2^{n}}\right]\right), i=0,1,2, \ldots, 2^{n}-1 ; \\
& m_{n, i}^{\prime}=\inf _{e s s}\left(f ;\left[\frac{i}{2^{n}}, \frac{i+1}{2^{n}}\right]\right), i=0,1,2, \ldots, 2^{n}-1 ; \\
& M_{n, i}^{\prime \prime}=\sup _{e s s}\left(f ;\left[\frac{2 i-1}{2^{n}}, \frac{2 i+1}{2^{n}}\right]\right) \quad i=1,2, \ldots, 2^{n-1}-1 ; \\
& m_{n, i}^{\prime \prime}=\inf _{e s s}\left(f ;\left[\frac{2 i-1}{2^{n}}, \frac{2 i+1}{2^{n}}\right]\right), i=1,2, \ldots, 2^{n-1}-1 ; \\
& A_{n, i}^{\prime}=\left\{x \in\left(\frac{i}{2^{n}}, \frac{i+1}{2^{n}}\right): m_{n, i}^{\prime} \leq f(x) \leq M_{n, i}^{\prime}\right\}, i=0,1,2, \ldots, 2^{n}-1 ; \\
& A_{n, i}^{\prime \prime}=\left\{x \in\left(\frac{2 i-1}{2^{n}}, \frac{2 i+1}{2^{n}}\right): m_{n, i}^{\prime \prime} \leq f(x) \leq M_{n, i}^{\prime \prime}\right\}, i=1,2, \ldots, 2^{n-1}-1 ; \\
& A_{n}^{\prime}=\cup_{i=1}^{2^{n}-1} A_{n, i}^{\prime} ; \\
& A_{n}^{\prime \prime}=A_{n, 0}^{\prime} \cup A_{n, 2^{n}-1}^{\prime} \cup\left(\cup_{i=1}^{2^{n-1}-1} A_{n, i}^{\prime \prime}\right) .
\end{aligned}
$$

For example, each set

$$
\begin{gathered}
\left\{x \in\left(\frac{i}{2^{n}}, \frac{i+1}{2^{n}}\right): f(x)>M_{n, i}^{\prime}\right\}= \\
\bigcup_{k=1}^{\infty}\left\{x \in\left(\frac{i}{2^{n}}, \frac{i+1}{2^{n}}\right): f(x) \geq M_{n, i}^{\prime}+\frac{1}{k}\right\},
\end{gathered}
$$

$i=1,2, \ldots 2^{n}-1$, is therefore a countable union of null sets. Thus $A_{n}^{\prime}, A_{n}^{\prime \prime}$ are measurable sets and $\left|A_{n}^{\prime}\right|=\left|A_{n}^{\prime \prime}\right|=1$. Let $A=\cap_{n=2}^{\infty}\left(A_{n}^{\prime} \cup A_{n}^{\prime \prime}\right)$. Then $A$ is measurable and $|A|=1$.

We show that $F \in V B$ on $A$. Since $F \in E V B$ on $[a, b]$, there exists $M>0$ such that

$$
\sum_{i=0}^{2^{n}-1} \mathcal{O}_{\text {ess }}\left(f ;\left[\frac{i}{2^{n}}, \frac{i+1}{2^{n}}\right]\right)=\sum_{i=0}^{2^{n}-1}\left(M_{n, i}^{\prime}-m_{n, i}^{\prime}\right)<M, n \geq 2 \text { and }
$$




$$
\sum_{i=1}^{2^{n-1}-1} \mathcal{O}_{\text {ess }}\left(f ;\left[\frac{2 i-1}{2^{n}}, \frac{2 i+1}{2^{n}}\right]\right)=\sum_{i=1}^{2^{n-1}-1}\left(M_{n, i}^{\prime \prime}-m_{n, i}^{\prime \prime}\right)<M, n \geq 2 .
$$

Let $\left\{\left[\alpha_{k}, \beta_{k}\right]\right\}, k=1,2, \ldots, p$ be a finite set of nonoverlapping closed intervals with endpoints in $A$. Then there exists a positive integer $n_{o}$ such that each $\alpha_{k}$ and $\beta_{k}$ is contained in the interior of exactly one component interval of the partition $\pi_{n_{o}}^{\prime}$. Let $x_{0}<x_{1}<x_{2}<\ldots<x_{2^{n_{o}-1}}$ be such that

$$
\begin{aligned}
& x_{i} \in A \cap\left(\frac{i}{2^{n_{o}}}, \frac{i+1}{2^{n_{o}}}\right), i=0,1, \ldots, 2^{n_{o}}-1, \text { and } \\
& \left\{x_{0}, x_{1}, \cdots x_{2^{n_{o}}-1}\right\} \supseteq\left\{\alpha_{1}, \beta_{1}, \alpha_{2}, \beta_{2} \cdots \alpha_{n}, \beta_{n}\right\} .
\end{aligned}
$$

Clearly

$$
\begin{gathered}
\sum_{k=1}^{p}\left|f\left(\beta_{k}\right)-f\left(\alpha_{k}\right)\right| \leq \sum_{k=1}^{p} \sum_{\left[x_{i-1}, x_{i}\right] \subseteq\left[\alpha_{k}, \beta_{k}\right]}\left|f\left(x_{i}\right)-f\left(x_{i-1}\right)\right| \leq \\
\sum_{i=1}^{2^{n_{o}}-1}\left|f\left(x_{i}\right)-f\left(x_{i-1}\right)\right| .
\end{gathered}
$$

But

$$
\sum_{i=0}^{2^{n_{o}}-1}\left|f\left(x_{2 i+1}\right)-f\left(x_{2 i}\right)\right|<\sum_{i=0}^{2^{n_{o}-1}-1}\left(M_{n_{o}-1, i}^{\prime}-m_{n_{o}-1, i}^{\prime}\right)<M
$$

(because $2 i / 2^{n_{o}}<x_{2 i}<(2 i+1) / 2^{n_{o}}<x_{2 i+1}<(2 i+2) / 2^{n_{o}}$, so $i /\left(2^{n_{o}-1}\right)<$ $\left.x_{2 i}<x_{2 i+1}<(i+1) /\left(2^{n_{o}-1}\right)\right)$ and

$$
\sum_{i=1}^{2^{n_{o}-1}-1}\left|f\left(x_{2 i}\right)-f\left(x_{2 i-1}\right)\right|<\sum_{i=1}^{2^{n_{o}-1}-1}\left(M_{n_{o}, i}^{\prime \prime}-m_{n_{o}, i}^{\prime \prime}\right)<M
$$

(because $\left.(2 i-1) / 2^{n_{o}}<x_{2 i-1}<2 i / 2^{n_{o}}<x_{2 i}<(2 i+1) / 2^{n_{o}}\right)$. It follows that $\sum_{k=1}^{p}\left|f\left(b_{k}\right)-f\left(a_{k}\right)\right|<2 M$, hence $f \in V B$ on $A$. Moreover $V(f ; A) \leq 2$. $E V(f ;[a, b])$. By Lemma 2 , it follows that there exists $\tilde{f}:[a, b] \rightarrow \mathbb{R}$ such that $\tilde{f}=f$ on $A$ and $V(\tilde{f} ;[a, b])=V(f ; A)$. Therefore $V(\tilde{f} ;[a, b]) \leq 2 \cdot E V(f ;[a, b])$.

(ii) $\Rightarrow(i)$ Let $M>0$ be given by the fact that $\tilde{f} \in V B$ on $[a, b]$. Let $\left\{\left[a_{i}, b_{i}\right]\right\}$, $i=1,2, \ldots, n$ be a set of nonoverlapping closed subintervals of $[a, b]$. Then $M>\sum_{i=1}^{n} \mathcal{O}\left(f ; A \cap\left[a_{i}, b_{i}\right]\right) \geq \sum_{i=1}^{n} \mathcal{O}_{\text {ess }}\left(f ;\left[a_{i}, b_{i}\right]\right)$. It follows that $f \in E V B$ on $[a, b]$. Moreover $E V(f ;[a, b]) \leq V(\tilde{f} ;[a, b])$. 
Remark 1. Lemma 2 is in fact an observation of [14] (p. 81). It was used for example in the proof of Sargent's Theorem 50 (see [3], p. 45) without demonstration, but with the warning of Peter Bullen (see [3], p. 309) that a more complete proof of it is in [16].

Definition 2. A function $F:[a, b] \rightarrow \mathbb{R}$ is said to be of bounded slope variation (abbreviated $F \in B S V$ ) on a subset $P$ of $[a, b]$, if there exists $M>0$ such that

$$
\begin{aligned}
\sum_{i=1}^{n} \mid & \left|\frac{F\left(b_{2 i}\right)-F\left(a_{2 i}\right)}{b_{2 i}-a_{2 i}}-\frac{F\left(b_{2 i-1}\right)-F\left(a_{2 i-1}\right)}{b_{2 i-1}-a_{2 i-1}}\right|<M \\
& \text { whenever } a_{1}<b_{1} \leq a_{2}<b_{2} \leq \ldots \leq a_{2 n}<b_{2 n} \text { are points in } P
\end{aligned}
$$

Let $S V(F ; P)=\inf \{M:(1)$ holds. $\}$ If $F \notin B S V$ on $P$ let $S V(F ; P)=$ $+\infty$

Lemma 3. Let $F:[a, b] \rightarrow \mathbb{R}$. The following assertions are equivalent:

(i) $F \in B S V$ on $[a, b]$.

(ii) There exists $M>0$ such that

$$
\sum_{i=0}^{n-2}\left|\frac{F\left(x_{i+2}\right)-F\left(x_{i+1}\right)}{x_{i+2}-x_{i+1}}-\frac{F\left(x_{i+1}\right)-F\left(x_{i}\right)}{x_{i+1}-x_{i}}\right|<M,
$$

whenever $a=x_{o}<x_{1}<x_{2}<\ldots<x_{n}=b$.

Proof. $(i) \Rightarrow($ ii $)$ Let $\mathrm{M}$ be given by the fact that $F \in B S V$ on $[a, b]$. We have

$$
\begin{aligned}
& \sum_{i=0}^{n-2}\left|\frac{F\left(x_{i+2}\right)-F\left(x_{i+1}\right)}{x_{i+2}-x_{i+1}}-\frac{F\left(x_{i+1}\right)-F\left(x_{i}\right)}{x_{i+1}-x_{i}}\right| \\
= & \sum_{\substack{i=0 \\
i=\text { even }}}^{n-2}\left|\frac{F\left(x_{i+2}\right)-F\left(x_{i+1}\right)}{x_{i+2}-x_{i+1}}-\frac{F\left(x_{i+1}\right)-F\left(x_{i}\right)}{x_{i+1}-x_{i}}\right| \\
= & \sum_{\substack{i=0 \\
i=0 \text { odd }}}^{n-2}\left|\frac{F\left(x_{i+2}\right)-F\left(x_{i+1}\right)}{x_{i+2}-x_{i+1}}-\frac{F\left(x_{i+1}\right)-F\left(x_{i}\right)}{x_{i+1}-x_{i}}\right|<M+M=2 M .
\end{aligned}
$$

(ii) $\Rightarrow\left(\right.$ i) We may suppose without loss of generality that $a<a_{1}<b_{1}<a_{2}<$ $b_{2}<\ldots<a_{2 n}<b_{2 n}<b$. Let's rename these points $a=x_{0}<x_{1}<x_{2}<$ 
$\ldots<x_{4 n+1}=b$. Then we have

$$
\begin{aligned}
& \sum_{i=1}^{n}\left|\frac{F\left(b_{2 i}\right)-F\left(a_{2 i}\right)}{b_{2 i}-a_{2 i}}-\frac{F\left(b_{2 i-1}\right)-F\left(a_{2 i-1}\right)}{b_{2 i-1}-a_{2 i-1}}\right| \\
\leq & \sum_{i=1}^{n}\left|\frac{F\left(b_{2 i}\right)-F\left(a_{2 i}\right)}{b_{2 i}-a_{2 i}}-\frac{F\left(a_{2 i}\right)-F\left(b_{2 i-1}\right)}{a_{2 i}-b_{2 i-1}}\right| \\
& +\sum_{i=1}^{n}\left|\frac{F\left(a_{2 i}\right)-F\left(b_{2 i-1}\right)}{a_{2 i}-b_{2 i-1}}-\frac{F\left(b_{2 i-1}\right)-F\left(a_{2 i-1}\right)}{b_{2 i-1}-a_{2 i-1}}\right| \\
\leq & \sum_{i=0}^{4 n-1}\left|\frac{F\left(x_{i+2}\right)-F\left(x_{i+1}\right)}{x_{i+2}-x_{i+1}}-\frac{F\left(x_{i+1}\right)-F\left(x_{i}\right)}{x_{i+1}-x_{i}}\right|<M .
\end{aligned}
$$

Remark 2. Lemma 3, (ii) is in fact Definition 12.5 of [12] (p. 74) for the condition $B S V$ on $[a, b]$.

Theorem 1. With the above notations we have the following results:

(i) Let $f:[a, b] \rightarrow \mathbb{R}, f \in E B V$ and let $F(x)=(\mathcal{L}) \int_{a}^{x} f(t) d t$. Then $F \in B S V$ on $[a, b]$ and $S V(F ;[a, b]) \leq E V(f ;[a, b])$.

(ii) Let $F:[a, b] \rightarrow \mathbb{R}, F \in B S V$ and let

$$
F^{*}(x)= \begin{cases}F^{\prime}(x) & \text { where } F \text { is derivable } \\ 0 & \text { elsewhere. }\end{cases}
$$

Then $F$ satisfies the Lipschitz condition $L, F^{*} \in E B V$ on $[a, b]$ and $E V\left(F^{*} ;[a, b]\right) \leq S V(F ;[a, b])$.

Proof. (i) Clearly $f$ is essentially bounded on $[a, b]$; so $f$ is summable on $[a, b]$ and $F(x)=(\mathcal{L}) \int_{a}^{x} f(t) d t$ is Lipschitz. Let $a \leq a_{1}<b_{1} \leq a_{2}<b_{2} \leq$ $\ldots \leq a_{2 n}<b_{2 n} \leq b$. We have

$$
\inf _{e s s}\left(f ;\left[a_{2 i-1}, b_{2 i}\right]\right) \leq \frac{F\left(b_{2 i}\right)-F\left(a_{2 i}\right)}{b_{2 i}-a_{2 i}} \leq \sup _{e s s}\left(f ;\left[a_{2 i-1}, b_{2 i}\right]\right)
$$

and

$$
\inf _{e s s}\left(f ;\left[a_{2 i-1}, b_{2 i}\right]\right) \leq \frac{F\left(b_{2 i-1}\right)-F\left(a_{2 i-1}\right)}{b_{2 i-1}-a_{2 i-1}} \leq \sup _{e s s}\left(f ;\left[a_{2 i-1}, b_{2 i}\right]\right) .
$$


Hence

$$
\left|\frac{F\left(b_{2 i}\right)-F\left(a_{2 i}\right)}{b_{2 i}-a_{2 i}}-\frac{F\left(b_{2 i-1}\right)-F\left(a_{2 i-1}\right)}{b_{2 i-1}-a_{2 i-1}}\right|<\mathcal{O}_{e s s}\left(f ;\left[a_{2 i-1}, b_{2 i}\right]\right) .
$$

Let $\epsilon>0$. Then

$$
\begin{gathered}
\sum_{i=1}^{n}\left|\frac{F\left(b_{2 i}\right)-F\left(a_{2 i}\right)}{b_{2 i}-a_{2 i}}-\frac{F\left(b_{2 i-1}\right)-F\left(a_{2 i-1}\right)}{b_{2 i-1}-a_{2 i-1}}\right|< \\
\sum_{i=1}^{n} \mathcal{O}_{e s s}\left(F ;\left[a_{2 i-1}, b_{2 i}\right)<(\epsilon+E V(f ;[a, b])) .\right.
\end{gathered}
$$

Hence $F \in B S V$ on $[a, b]$. Since $\epsilon$ was arbitrary, $S V(F ;[a, b]) \leq E V(f ;[a, b])$.

(ii) We show that $F$ is bounded on $[a, b]$. Suppose for example that $F$ is upper unbounded. Then there exists a sequence $\left\{x_{n}\right\}_{n}$ such that $F\left(x_{n}\right)>n$ for each $n$. For $F\left(x_{n}\right)>\max \{|F(a)|,|F(b)|\}$ we have

$$
\left|\frac{F(b)-F\left(x_{n}\right)}{b-x_{n}}-\frac{F\left(x_{n}\right)-F(a)}{x_{n}-a}\right|>\frac{F\left(x_{n}\right)-F(a)}{x_{n}-a}>\frac{n-a}{b-a} \rightarrow+\infty .
$$

Hence $F \notin B S V$ on $[a, b]$, a contradiction.

Suppose on the contrary that $F \notin L$ on $[a, b]$. For each positive integer $n$, there exist $x_{n}, y_{n} \in[a, b], x_{n}<y_{n}$, such that $\left|F\left(y_{n}\right)-F\left(x_{n}\right)\right| /\left(y_{n}-x_{n}\right)>n$. Since $F$ is bounded, $y_{n}-x_{n} \rightarrow 0$. But $\left\{x_{n}\right\}_{n}$ is a bounded sequence; so it contains a convergent subsequence. Hence, we may suppose without loss of generality that $\left\{x_{n}\right\}_{n}$ converges to $x_{o}$. Then $\left\{y_{n}\right\}_{n}$ converges to $x_{o}$ too. We have two cases:

1) If $x_{o}=a$, then there exists $n_{o}$ such that $y_{n}<(a+b) / 2$ for each $n \geq n_{o}$. It follows that $\left[x_{n}, y_{n}\right]$ and $[(a+b) / 2, b]$ are nonoverlapping closed intervals for each $n \geq n_{o}$. We have

$$
\left|\frac{F(b)-F((a+b) / 2)}{(b-a) / 2}-\frac{F\left(y_{n}\right)-F\left(x_{n}\right)}{y_{n}-x_{n}}\right| \rightarrow+\infty, \quad n \rightarrow \infty .
$$

This contradicts the fact that $F \in B S V$ on $[a, b]$.

2) If $x_{o} \neq a$, then there exists $n_{o}$ such that $x_{n}>\left(a+x_{o}\right) / 2$, for each $n>n_{o}$. It follows that $\left[a,\left(a+x_{o}\right) / 2\right]$ and $\left[x_{n}, y_{n}\right]$ are nonoverlapping closed intervals for each $n \geq n_{o}$. We have

$$
\left|\frac{F\left(y_{n}\right)-F\left(x_{n}\right)}{y_{n}-x_{n}}-\frac{F\left(\left(a+x_{o}\right) / 2\right)-F(a)}{\left(x_{o}-a\right) / 2}\right| \rightarrow+\infty, \quad n \rightarrow \infty .
$$


This contradicts the fact that $F \in B S V$ on $[a, b]$.

Therefore we have obtained that $F \in L$ on $[a, b]$. It follows that $\mathrm{F}$ is derivable a.e. on $[a, b]$. Let $A=\{x \in[a, b]: F$ is derivable at $x\}$. Clearly $F^{*}=F^{\prime}$ on $A$. We show that $F^{\prime} \in V B$ on $A$. Let $a_{1}<b_{1} \leq a_{2}<b_{2} \leq \ldots \leq$ $a_{n}<b_{n}$ be points in $A$. For $\epsilon>0$ let $\left[c_{i}, d_{i}\right] \subset\left(a_{i}, b_{i}\right)$ such that

$$
\left|\frac{F\left(c_{i}\right)-F\left(a_{i}\right)}{c_{i}-a_{i}}-F^{\prime}\left(a_{i}\right)\right|<\frac{\epsilon}{2 n} \text { and }\left|\frac{F\left(b_{i}\right)-F\left(d_{i}\right)}{b_{i}-d_{i}}-F^{\prime}\left(b_{i}\right)\right|<\frac{\epsilon}{2 n} \text {. }
$$

We have

$$
\begin{aligned}
& \sum_{i=1}^{n}\left|F^{\prime}\left(b_{i}\right)-F^{\prime}\left(a_{i}\right)\right| \leq \sum_{i=1}^{n}\left|\frac{F\left(c_{i}\right)-F\left(a_{i}\right)}{c_{i}-a_{i}}-F^{\prime}\left(a_{i}\right)\right| \\
& +\sum_{i=1}^{n}\left|\frac{F\left(b_{i}\right)-F\left(d_{i}\right)}{b_{i}-d_{i}}-F^{\prime}\left(b_{i}\right)\right|+\sum_{i=1}^{n}\left|\frac{F\left(c_{i}\right)-F\left(a_{i}\right)}{c_{i}-a_{i}}-\frac{F\left(b_{i}\right)-F\left(d_{i}\right)}{b_{i}-d_{i}}\right| \\
< & \frac{\epsilon}{2}+\frac{\epsilon}{2}+(\epsilon+S V(F ;[a, b])) .
\end{aligned}
$$

Therefore $F^{*} \in V B$ on $A$. Since $\epsilon$ was arbitrary, $V\left(F^{*} ; A\right) \leq S V(F ;[a, b])$. Let $\left\{\left[a_{i}, b_{i}\right]\right\}, i=1,2, \cdots n$ be a set of nonoverlapping closed intervals of $[a, b]$. Then $V\left(F^{*} ; A\right) \geq \sum_{i=1}^{n} \mathcal{O}\left(F^{*} ; A \cap\left[a_{i}, b_{i}\right]\right) \geq \sum_{i=1}^{n} \mathcal{O}_{\text {ess }}\left(F^{*} ;\left[a_{i}, b_{i}\right]\right)$. Therefore $E V\left(F^{*} ;[a, b]\right) \leq S V(F ;[a, b])$.

Corollary 1. A function $F:[a, b] \rightarrow \mathbb{R}$ is the indefinite Lebesgue integral of a $V B$ function $f:[a, b] \rightarrow \mathbb{R}$, if and only if $F \in B V S$ on $[a, b]$.

Proof. See Theorem 1 and Lemma 2.

Remark 3. If in Corollary 1 " $F \in B V S$ " is replaced by " $F \in B S V \cap L$ " we obtain a result of Riesz (Lemma 12.6 of [12], p.75). As we see from our Theorem 1 " $F \in B S V \cap L$ " is superfluous, because " $B S V \in L$ ". Let's mention that in the prove of Theorem 1 we used some techniques of Riesz' lemma.

\section{A General Descriptive definition for Integration}

Definition 3. A class of functions $\mathcal{P}([a, b]) \subset\{F:[a, b] \rightarrow \mathbb{R}: F$ is continuous on $[a, b]$ and approximately derivable $a . e$. on $[a, b]\}$ is called a general class of primitives on $[a, b]$ if it satisfies the following properties :

(i) $\mathcal{P}([a, b])$ is a real linear space ;

(ii) If $F_{a p}^{\prime}=G_{a p}^{\prime}$ a.e. on $[a, b]$ and $F, G \in \mathcal{P}([a, b])$, then $F-G$ is a constant on $[a, b]$; 
(iii) If $F \in \mathcal{P}([a, b])$ and $g:[a, b] \rightarrow \mathbb{R}$ is a $V B$ function on $[a, b]$, then $H \in \mathcal{P}([a, b])$, where $H(x)=F(x) \cdot g(x)-(\mathcal{R S}) \int_{a}^{x} F(t) d g(t)$ and $(\mathcal{R S})$ stands for the Riemann-Stieltjes integral.

(iv) $\mathcal{P}([a, b])$ contains the class $\operatorname{Lip}([a, b])=\{F:[a, b] \rightarrow \mathbb{R}: F$ is Lipschitz $\}$.

Definition 4. A function $f:[a, b] \rightarrow \overline{\mathbb{R}}$ is said to be $\mathcal{P}$-integrable on $[a, b]$ if there exists a function $F:[a, b] \rightarrow \mathbb{R}$ such that $F_{a p}^{\prime}(x)=f(x)$ a.e. on $[a, b]$. We will write $(\mathcal{P}) \int_{a}^{b} f(t) d t=F(b)-F(a)$. We refer to $F$ as $\mathcal{P}$-primitive of $f$ on $[a, b]$.

Remark 4. Note the following:

(i) From Definition 3 (ii) it follows that the $\mathcal{P}$-integral is well defined.

(ii) By Definition 3 (i) it follows that the set of all $\mathcal{P}$-integrable functions on $[a, b]$ is a real linear space.

(iii) If $f:[a, b] \rightarrow \mathbb{R}$ is $\mathcal{P}$-integrable, then $f$ is measurable (see [15], p. 299).

(iv) We will define on the set of all $\mathcal{P}$-integrable functions on $[a, b]$ an equivalence relation : $f \sim g$ if $f(x)=g(x)$ a.e. on $[a, b]$.

(v) We denote the set of all classes of equivalence with $\mathcal{P}_{\text {int }}([a, b])$. With the usual operations with classes the set $\mathcal{P}_{\text {int }}([a, b])$ becomes a real linear space. We shall denote the equivalence class of $f$ also by $f$.

(vi) Let $\mathcal{P}_{o}([a, b])=\{F:[a, b] \rightarrow \mathbb{R}: F \in \mathcal{P}([a, b]), F(a)=0\}$.

(vii) Formula $\|F\|_{\infty}=\sup _{x \in[a, b]}|F(x)|$ defines a norm on each of the following linear spaces: $\mathcal{P}_{o}([a, b]), \mathcal{P}([a, b]), C([a, b]), C_{o}([a, b])$ (here $C([a, b])=$ $\{f:[a, b] \rightarrow \mathbb{R}: f$ is continuous $\}$ and $C_{o}([a, b])=\{f:[a, b] \rightarrow \mathbb{R}: f$ is continuous and $f(a)=0\})$.

(viii) Let $f \in \mathcal{P}_{\text {int }}([a, b])$ and let $F \in \mathcal{P}_{o}([a, b])$ be the unique $\mathcal{P}$ primitive of $f$. The formula $\|f\|=\|F\|_{\infty}$ defines a norm on $\mathcal{P}_{\text {int }}([a, b])$.

(ix) We denote by $V B([a, b])=\{g:[a, b] \rightarrow \mathbb{R}: g \in V B$ on $[a, b]\}$. With the usual operations with functions and with the norm $\|g\|_{V B}=|g(b)|+$ $V_{a}^{b}(g)$, the set $V B([a, b])$ becomes a real Banach space. 


\section{Riesz representation theorems for the $\mathcal{P}$ integration}

Definition 5. Let $\langle\cdot, \cdot\rangle: \mathcal{P}_{\text {int }}([a, b]) \times V B([a, b]) \rightarrow \mathbb{R}$ be defined by the formula $\langle f, g\rangle=(\mathcal{P}) \int_{a}^{b} f(t) g(t) d t$. (That $f \cdot g$ is $\mathcal{P}$-integrable on $[a, b]$ follows by Definition 3 (iii) and the fact that $H_{a p}^{\prime}(x)=f(x) g(x)$ a.e. on $[a, b]$, see the proof of Theorem 5.23 .2 of [5].)

Lemma 4. Let $f \in \mathcal{P}_{\text {int }}([a, b])$ and $g \in V B([a, b])$. Then we have :

(i) $\langle\cdot, \cdot\rangle$ is bilinear

(ii) $|\langle f, g\rangle| \leq\|f\| \cdot\|g\|_{V B}$

(iii) $T: \mathcal{P}_{\text {int }}([a, b]) \rightarrow \mathbb{R}, T(f)=\langle f, g\rangle$ is a continuous linear functional and $\|T\| \leq\|g\|_{V B}$.

Proof. By Definitions 3 and $5,\langle f, g\rangle=F(b) g(b)-(\mathcal{R S}) \int_{a}^{b} F(t) d g(t)$, where $F \in \mathcal{P}_{o}([a, b])$ is the unique $\mathcal{P}$-primitive of $f$.

(i) This follows by the fact that the $\mathcal{R S}$-integral is linear in the first argument and in the second argument.

(ii) We have $|\langle f, g\rangle|=\left|F(b) g(b)-(\mathcal{R S}) \int_{a}^{b} F(t) d g(t)\right| \leq|F(b)| \cdot|g(b)|+$ $\|F\|_{\infty} \cdot V(g ;[a, b]) \leq\|F\|_{\infty} \cdot(|g(b)|+V(g ;[a, b]))=\|f\| \cdot\|g\|_{V B}$.

(iii) This follows by (i) and (ii).

Lemma 5. Let $\left(X,\|\cdot\|_{1}\right)$ and $\left(Y,\|\cdot\|_{2}\right)$ be normed real spaces and let $\langle\cdot, \cdot\rangle$ : $X \times Y \rightarrow \mathbb{R}$ be such that:

a) $\langle\cdot, y\rangle$ is linear in the first variable, for each $y \in Y$;

b) $|\langle x, y\rangle| \leq\|x\|_{1} \cdot\|y\|_{2}$, whenever $x \in X, y \in Y$.

If $f: X \rightarrow \mathbb{R}$ is a continuous linear functional and if there exist $y_{o} \in Y$ and a dense subset $X_{o}$ of $X$ such that $f(x)=\left\langle x, y_{o}\right\rangle$ for each $x \in X_{o}$, then $f(x)=\left\langle x, y_{o}\right\rangle$ on $X$ and $\|f\| \leq\left\|y_{o}\right\|_{2}$.

Proof. Since $\bar{X}_{o}=X$, for $x \in X$ there exists a sequence $\left\{x_{n}\right\}_{n} \subset X_{o}$ such that $\left\|x_{n}-x\right\|_{1} \rightarrow 0$, for $n \rightarrow \infty$. But $\left|\left\langle x_{n}, y_{o}\right\rangle-\left\langle x, y_{o}\right\rangle\right|=\left|\left\langle x_{n}-x, y_{o}\right\rangle\right| \leq \| x_{n}-$ $x\left\|_{1} \cdot\right\| y_{o} \|_{2}$ (see a)and b)). Since $f$ is continuous, $f(x)=\lim _{n \rightarrow \infty}\left\langle x_{n}, y_{o}\right\rangle=$ $\left\langle x, y_{o}\right\rangle$. Hence $f(x)=\left\langle x, y_{o}\right\rangle$, for each $x \in X$ and $\|f\| \leq\left\|y_{o}\right\|_{2}$ (see a) and b)).

Theorem 2. Let $T: \mathcal{P}_{\text {int }}([a, b]) \rightarrow \mathbb{R}$ be a continuous linear functional. Then there exists $g \in V B$ such that

$$
T(f)=\langle f, g\rangle=(\mathcal{P}) \int_{a}^{b} f(t) g(t) d t \quad \text { and }
$$




$$
\frac{1}{2} V(g ;[a, b]) \leq\|L\| \leq\|g\|_{V B}
$$

ProOF. Let

$$
\begin{aligned}
\mathcal{S}([a, b])= & \{s:[a, b] \rightarrow \mathbb{R}: s \text { is a step function of the form } s(t)= \\
& \sum_{i=1}^{n-1} \alpha_{i} K_{\left[t_{i-1}, t_{i}\right)}+\alpha_{n} K_{\left[t_{n-1}, t_{n}\right]} \text { for some positive integer } n, \\
& \text { where each } \left.\alpha_{i} \in \mathbb{R}, a=t_{0}<t_{1}<\ldots<t_{n}=b\right\} .
\end{aligned}
$$

(Here $K_{E}$ denotes the characteristic function of the set $E$.) We show that $\overline{\mathcal{S}([a, b])}=\mathcal{P}_{\text {int }}([a, b])$. Let $f \in \mathcal{P}_{\text {int }}([a, b])$ and let $F \in \mathcal{P}_{o}([a, b]$ the unique primitive of $f$. Then $F(x)$ is continuous on $[a, b]$. Let $a=x_{0}<x_{1}<\ldots<$ $x_{n}=b, x_{i}-x_{i-1}=(b-a) / n$ for each $i=1,2, \ldots, n$. Let $F_{n}\left(x_{i}\right)=F\left(x_{i}\right)$, $i=0,1, \ldots, n$ and let $F_{n}$ be linear on each closed interval $\left[x_{i-1}, x_{i}\right]$. Then $F_{n} \stackrel{[u n i f]}{\longrightarrow} F$ on $[a, b]$ and each $F_{n}$ is Lipschitz. By Definition 3 (iv), each $F_{n}$ is in $\mathcal{P}_{o}([a, b])$. Let

$$
s_{n}(x)= \begin{cases}\frac{F\left(x_{i}\right)-F\left(x_{i-1}\right)}{x_{i}-x_{i-1}} & \text { if } x \in\left[x_{i-1}, x_{i}\right), i=1,2, \ldots, n-1 \\ \frac{F\left(x_{n}\right)-F\left(x_{n-1}\right)}{x_{n}-x_{n-1}} & \text { if } x \in\left[x_{n-1}, x_{n}\right]\end{cases}
$$

Then $s_{n} \in \mathcal{S}([a, b])$ and $\left\|s_{n}-f\right\|=\left\|F_{n}-F\right\|_{\infty} \rightarrow 0$ (because $F_{n} \stackrel{[\text { unif] }}{\longrightarrow}$ on $[a, b])$. Therefore $\mathcal{S}([a, b])$ is dense in $\mathcal{P}_{\text {int }}([a, b])$.

Let $G(t)=T\left(K_{[a, t]}\right)$ and let $a \leq a_{1}<b_{1} \leq a_{2}<b_{2} \leq \ldots \leq a_{2 n}<b_{2 n} \leq b$. Since $T$ is linear and continuous,

$$
\begin{gathered}
\sum_{i=1}^{n}\left|\frac{G\left(b_{2 i}\right)-G\left(a_{2 i}\right)}{b_{2 i}-a_{2 i}}-\frac{G\left(b_{2 i-1}\right)-G\left(a_{2 i-1}\right)}{b_{2 i-1}-a_{2 i-1}}\right|= \\
\sum_{i=1}^{n}\left|T\left(\varphi_{i}\right)\right|=\sum_{i=1}^{n} \epsilon_{i} T\left(\varphi_{i}\right)=T\left(\sum_{i=1}^{n} \epsilon \varphi_{i}\right) \leq\|T\| \cdot\left\|\sum_{i=1}^{n} \epsilon_{i} \varphi_{i}\right\| \leq\|T\|
\end{gathered}
$$

where $\epsilon_{i}=\operatorname{sign} T\left(\varphi_{i}\right)$ and

$$
\varphi_{i}=\frac{1}{b_{2 i}-a_{2 i}} \cdot K_{\left(a_{2 i}, b_{2 i}\right]}-\frac{1}{b_{2 i-1}-a_{2 i-1}} \cdot K_{\left(a_{2 i-1}, b_{2 i-1}\right]} .
$$

It follows that $G \in B S V$ and

$$
S V(G ;[a, b]) \leq\|T\| .
$$


By Theorem 1, (ii) $G^{*} \in E B V$ and

$$
E V\left(G^{*},[a, b]\right) \leq S V(G ;[a, b]) .
$$

By Lemma 2 it follows that there exists a function $g:[a, b] \rightarrow \mathbb{R}$ such that $g \in V B, g=G^{*}$ a.e. on $[\mathrm{a}, \mathrm{b}]$ and

$$
E V\left(G^{*} ;[a, b]\right) \leq V(g ;[a, b]) \leq 2 \cdot E V\left(G^{*} ;[a, b]\right)
$$

Clearly $G(t)=(\mathcal{L}) \int_{a}^{t} G^{*}(x) d x=(\mathcal{L}) \int_{a}^{b} K_{[a, t]}(x) G^{*}(x) d x=T\left(K_{[a, t]}\right)$. Since $T$ is linear, it follows that $T(s)=\langle s, g\rangle$ whenever $s \in \mathcal{S}([a, b])$. By Lemma 5 we have $T(f)=\langle f, g\rangle$ for every $f \in \mathcal{P}_{\text {int }}([a, b])$ and $\|T\| \leq\|g\|_{V B}$. By (5) and (4), $E V\left(G^{*} ;[a, b]\right) \leq\|T\|$. Hence $E V\left(G^{*} ;[a, b]\right) \leq\|T\| \leq\|g\|_{V B}$. Now by $(6)$ it follows that $\frac{1}{2} \cdot V(g ;[a, b]) \leq\|T\| \leq\|g\|_{V B}$

Remark 5. Theorem 2 extends Alexiewicz' Theorem 12.7 of [12] (see also [1]).

Lemma 6. The normed spaces $\left(\mathcal{P}_{\text {int }}([a, b]),\|\cdot\|\right)$ and $\left(\mathcal{P}_{o}([a, b]),\|\cdot\|_{\infty}\right)$ are isomorph.

Proof. Let $\Phi:\left(\mathcal{P}_{\text {int }}([a, b]) \rightarrow\left(\mathcal{P}_{o}([a, b]), \Phi(f)=F\right.\right.$ where $F$ is the unique $\mathcal{P}$-primitive of $f$ which is contained in $\left(\mathcal{P}_{o}([a, b])\right.$. It is easy to verify that $\Phi$ is well defined, linearly, bijective and $\|\Phi(f)\|_{\infty}=\|f\|$

Lemma 7. We have the following results:

(i) The completion of $\left(\mathcal{P}([a, b]) ;\|\cdot\|_{\infty}\right)$ is $\left(C([a, b]),\|\cdot\|_{\infty}\right)$.

(ii) The completion of the isomorphic spaces $\left(\mathcal{P}_{\text {int }}([a, b]),\|\cdot\|\right)$ and $\left(\mathcal{P}_{o}([a, b])\right.$, $\left.\|\cdot\|_{\infty}\right)$ is $\left(C_{o}([a, b]),\|\cdot\|\right)$.

Proof. We prove only (ii). Let $F \in C_{o}([a, b])$. By the Weierstrass Theorem, there exists a sequence $\left\{P_{n}\right\}_{n}$ of polynomials on $[a, b]$ such that $\left\|P_{n}-F\right\|_{\infty} \rightarrow 0$ if $n \rightarrow \infty$. Let $Q_{n}(x)=P_{n}(x)-P_{n}(a)$. Then for each $n, Q_{n}(a)=0, Q_{n}$ is Lipschitz (hence $\left.Q_{n} \in \mathcal{P}_{o}([a, b])\right)$, and $\left\|Q_{n}-F\right\|_{\infty} \rightarrow 0$.

Remark 6. In the proof of Lemma 7 instead of $Q_{n}$ we can use $B_{n}$ the Bernstein polynomial of degree $n$ for the function $F$ on $[a, b]$, i.e.,

$$
B_{n}(x)=\sum_{k=0}^{n} F\left(a+\frac{k}{n}(b-a)\right) C_{n}^{k}\left(\frac{x-a}{b-a}\right)^{k}\left(\frac{b-x}{b-a}\right)^{n-k}
$$

(see [13], Definition 1 and Theorem 1, p. 108 and the proof of Theorem 2, p. 109). 
Theorem 3. We have the following results:

(i) Let $T:\left(\mathcal{P}_{o}([a, b]),\|\cdot\|_{\infty}\right) \rightarrow \mathbb{R}$ be a continuous linear functional. Then there exists $g \in V B$ on $[a, b]$ such that $T(F)=(\mathcal{R S}) \int_{a}^{b} F(t) d g(t)$, whenever $F \in \mathcal{P}_{o}([a, b])$.

(ii) Assertion (i) remains true if $\mathcal{P}_{o}([a, b])$ is replaced by $\mathcal{P}([a, b])$.

Proof. (i) Let $T^{*}: \mathcal{P}_{\text {int }}([a, b]) \rightarrow \mathbb{R}, T^{*}=T \circ \Phi$, where $\Phi$ is the isomorphicism defined in the proof of Lemma 6 . Since $T$ is a continuous linear functional, it follows that $T^{*}$ is also a continuous linear functional. By Theorem 2, there exists $G:[a, b] \rightarrow \mathbb{R}, G \in V B$, such that $T^{*}(f)=(\mathcal{P}) \int_{a}^{b} f(t) G(t) d t$. Let $F \in \mathcal{P}_{o}([a, b])$ and $f=\Phi^{-1}(F)$. Then

$$
\begin{aligned}
& T(F)=T^{*}(f)=(\mathcal{P}) \int_{a}^{b} f(t) G(t) d t=F(b) G(b)-(\mathcal{R S}) \int_{a}^{b} F(t) d G(t) \\
= & G(b) \cdot(\mathcal{R S}) \int_{a}^{b} F(t) d K_{\{b\}}(t)-(\mathcal{R S}) \int_{a}^{b} F(t) d G(t)=(\mathcal{R S}) \int_{a}^{b} F(t) d g(t)
\end{aligned}
$$

, where $g(t)=G(b) \cdot K_{\{b\}}(t)-G(t)$ (clearly $\left.g \in V B\right)$.

(ii) Let $\mathbb{I}:[a, b] \rightarrow \mathbb{R}, \mathbb{I}(x)=1$. Let $F \in \mathcal{P}([a, b])$ and $F_{o}(x)=F(x)-$ $F(a) \cdot \mathbb{I}(x)$. Then $F_{o} \in \mathcal{P}_{o}([a, b])$. By $(\mathrm{i})$,

$$
\begin{aligned}
& T(F)=T\left(F_{o}\right)+F(a) \cdot T(\mathbb{I})=(\mathcal{R S}) \int_{a}^{b} F_{o}(t) d g(t)+F(a) T(\mathbb{I}) \\
= & (\mathcal{R S}) \int_{a}^{b} F(t) d g(t)-F(a)(g(b)-g(a)-T(\mathbb{I})) \\
= & (\mathcal{R S}) \int_{a}^{b} F(t) d g(t)+(g(b)-g(a)-T(\mathbb{I})) \cdot(\mathcal{R S}) \int_{a}^{b} F(t) d K_{\{a\}}(t) \\
= & (\mathcal{R S}) \int_{a}^{b} F(t) d G(t),
\end{aligned}
$$

where $G(x)=g(x)+(g(b)-g(a)-T(\mathbb{I})) \cdot K_{\{a\}}(x)$ (clearly $\left.G \in V B\right)$.

Corollary 2 (The Riesz Representation Theorem [12]).

Let $T:\left(C([a, b]),\|\cdot\|_{\infty}\right) \rightarrow \mathbb{R}$ be a continuous linear functional. Then there exists $g \in V B$ on $[a, b]$ such that $T(F)=(\mathcal{R S}) \int_{a}^{b} F(t) d g(t)$, whenever $F \in$ $C([a, b])$.

Proof. Since $\mathcal{P}([a, b])$ is dense in $C([a, b])$ (see for example Lemma 7 (i)), for each $F \in C([a, b])$ there exists a sequence $\left\{F_{n}\right\}_{n}, F_{n} \in \mathcal{P}([a, b])$, such 
that $F_{n} \stackrel{[u n i f]}{\longrightarrow} F$ on $[a, b]$. Applying the Uniform Convergence Theorem for the $\mathcal{R S}$-integral and Theorem 3 (ii), we obtain $T(F)=\lim _{n \rightarrow \infty} T\left(F_{n}\right)=$ $\lim _{n \rightarrow \infty}(\mathcal{R S}) \int_{a}^{b} F_{n}(t) d g(t)=(\mathcal{R S}) \int_{a}^{b} F(t) d g(t)$.

Remark 7. In Corollary 1 we may replace the linear space $\left(C([a, b]),\|\cdot\|_{\infty}\right)$ by $\left(C_{o}([a, b]),\|\cdot\|_{\infty}\right)$.

\section{$5 \quad$ The Category of $\mathcal{P}_{\text {int }}([a, b])$}

Lemma 8. ([7], p. 49). Let $(X, \tau)$ be a topological space and let $X_{o}$ be a dense subset of $X$. Let $\tau_{o}=\tau_{\mid X_{o}}$. If $X_{o}$ is of the second category in $\left(X_{o}, \tau_{o}\right)$, then $X_{o}$ is of the second category in $(X, \tau)$.

Lemma 9 (Jarnik). ([2], p.224). Let $\left(C([a, b]),\|\cdot\|_{\infty}\right)$ and let $\mathcal{A}=\{f$ : $[a, b] \rightarrow \mathbb{R}: f$ is continuous and $f$ is nowhere approximately differentiable $\}$. Then $C([a, b]) \backslash \mathcal{A}$ is of the first category in $C([a, b])$.

Theorem 4. We have the following results:

(i) $\left(\mathcal{P}_{o}([a, b]),\|\cdot\|_{\infty}\right)$ is of first category on itself.

(ii) $\left(\mathcal{P}_{\text {int }}([a, b]),\|\cdot\|\right.$ is of first category on itself.

Proof. It suffices to prove only (ii) (because the proof of (i) is contained in (ii)). Suppose to the contrary that $\left(\mathcal{P}_{\text {int }}([a, b]),\|\cdot\|\right)$ is of the second category in itself. Since the spaces $\left(\mathcal{P}_{\text {int }}([a, b]),\|\cdot\|\right)$ and $\left(\mathcal{P}_{o}([a, b]),\|\cdot\|_{\infty}\right)$ are isomorphic (see Lemma 6), they are also homeomorphic. It follows that $\left(\mathcal{P}_{o}([a, b]),\|\cdot\|_{\infty}\right)$ is of the second category in itself. By Lemma 7 (ii), $\mathcal{P}_{o}([a, b])$ is dense in $C_{o}([a, b])$. By Lemma $8,\left(\mathcal{P}_{o}([a, b]),\|\cdot\|_{\infty}\right)$ is of second category in $\left(C_{o}([a, b]),\|\cdot\|_{\infty}\right)$, and by Lemma $9, \mathcal{P}_{o}([a, b])$ is of first category in $\left(C_{o}([a, b]),\|\cdot\|_{\infty}\right)$. This contradicts the fact that $\left(C_{o}([a, b]),\|\cdot\|_{\infty}\right)$ is a Banach space.

\section{Weak Convergence in $\mathcal{P}_{\text {int }}([a, b])$}

Theorem 5. ([11], p. 259). Let $f, f_{n}:[a, b] \rightarrow \mathbb{R}, n=1,2, \ldots$ be such that $f, f_{n}$ are continuous and $\left|f_{n}(x)\right|<M$ for some $M$, for every $x \in[a, b]$ and each $n=1,2, \ldots$. Let $g:[a, b] \rightarrow \mathbb{R}, g \in V B$. If $f_{n} \rightarrow f$ on $[a, b]$, then $(\mathcal{R S}) \int_{a}^{b} f(t) d g(t)=\lim _{n \rightarrow \infty}(\mathcal{R S}) \int_{a}^{b} f_{n}(t) d g(t)$.

Lemma 10 ([4] or [10], Theorem 2, \# 1 of Chapter VIII).

$x_{n} \rightarrow x$ weakly in a normed space if and only if $\sup _{n}\left\|x_{n}\right\|<+\infty$ and $\{f$ : $\left.f\left(x_{n}\right) \rightarrow f(x)\right\}$ is a dense set of functionals in $X^{*}$. 
Theorem 6. Let $f, f_{n} \in \mathcal{P}_{\text {int }}([a, b])$, and let $F, F_{n} \in \mathcal{P}_{o}([a, b])$ be the unique $\mathcal{P}$-primitives of $f, f_{n}, n=1,2, \ldots$ The following assertions are equivalent:

(i) $f_{n} \rightarrow f$ weakly on $\left(\mathcal{P}_{\text {int }}([a, b]),\|\cdot\|\right)$;

(ii) 1) $\left|F_{n}(x)\right| \leq M$ for some $M$, for every $x \in[a, b]$ and each $n=1,2, \ldots$;

2) $F_{n}(x) \rightarrow F(x)$ for every $x \in[a, b]$.

Proof. (i) $\Rightarrow$ (ii) Since $f_{n} \rightarrow f$ weakly, by Lemma 10 we obtain $\left\|f_{n}\right\|=$ $\left\|F_{n}\right\|_{\infty} \leq M$ for some positive number $M$. So we have 1) of (ii). For $x \in[a, b]$ let $T_{x}: \mathcal{P}_{\text {int }}([a, b]) \rightarrow \mathbb{R}$ be a continuous linear functional defined by $T_{x}(f)=$ $F(x)$ (because clearly $T_{x}$ is linear and $\left|T_{x}(f)\right|=|F(x)| \leq\|F\|_{\infty}=\|f\|$ ). Since $f_{n} \rightarrow f$ weakly it follows that $T_{x}\left(f_{n}\right) \rightarrow T_{x}(f)$, hence $F_{n}(x) \rightarrow F(x)$. Therefore we have condition 2) of (ii).

(ii) $\Rightarrow$ (i) Let $T: \mathcal{P}_{\text {int }}([a, b]) \rightarrow \mathbb{R}$ be a continuous linear functional. By Theorem 2 there exists $g_{T} \in V B$ on $[a, b]$ such that $T(f)=(\mathcal{P}) \int_{a}^{b} f(t) g_{T}(t) d t$, for every $f \in \mathcal{P}_{\text {int }}([a, b])$. We show that $T\left(f_{n}\right) \rightarrow T(f)$. Indeed, $\mid T\left(f_{n}\right)-$ $T(f)|=|(\mathcal{P}) \int_{a}^{b}\left(f_{n}-f\right)(t) g_{T}(t) d t|=|\left(F_{n}-F\right)(b) \cdot g_{T}(b)-(\mathcal{R S}) \int_{a}^{b}\left(F_{n}-\right.$ $F)(t) d g_{T}(t) \mid \rightarrow 0$ (see Theorem 5 ). Therefore we have (i).

Remark 8. We observe the following:

(i) Our proof parallels the proof of Theorem 3, \# 3, Chapter VIII of [10].

(ii) Using Theorem 3 (i) (respectively Remark 7) instead of Theorem 2 and $T_{x}: \mathcal{P}_{o}([a, b]) \rightarrow \mathbb{R}\left(\right.$ respectively $\left.T_{x}: C_{o}([a, b]) \rightarrow \mathbb{R}\right), T_{x}(F)=F(x)$, we can prove also the following theorem .

Let $F, F_{n} \in\left(\mathcal{P}_{o}([a, b]),\|\cdot\|_{\infty}\right)$ (respectively $\left(C_{o}([a, b]),\|\cdot\|_{\infty}\right) n=1,2, \cdots$. Then $F_{n} \rightarrow F$ weakly if and only if $\left|F_{n}(t)\right|<M$ for some $M$, whenever $t \in[a, b], n=1,2, \cdots$ and $F_{n}(t) \rightarrow F(t)$ for every $t \in[a, b]$.

\section{Applications}

In what follows we shall use the definitions given in [5] for the following classes of functions: $\mathcal{C}, A C, A C^{*}, A C_{n}, S A C_{n}, A C^{*} G, S A C G, \mathcal{F}, S \mathcal{F}$. We set

$$
\begin{aligned}
\Delta_{a e} & =\{F:[a, b] \rightarrow \mathbb{R}: F \text { is derivable a.e. on }[a, b]\} \Delta_{a p a e} \\
& =\{F:[a, b] \rightarrow \mathbb{R}: F \text { is approximately derivable a.e. on }[a, b]\} .
\end{aligned}
$$

Lemma 11 (A slight reformulation of Lemma 1 of [3], p. 31). Let $g:[a, b] \rightarrow$ $\mathbb{R}, g \in V B$, and let $F:[a, b] \rightarrow \mathbb{R}$ be a bounded function which is $\mathcal{R S}$ integrable on $[a, b]$ with respect to $g$. Let $H:[a, b] \rightarrow \mathbb{R}, H(x)=F(x) g(x)-$ $(\mathcal{R S}) \int_{a}^{x} F(t) d g(t)$. Then 
(i) $|H(\beta)-H(\alpha)| \leq \sup _{x \in[a, b]}|g(x)| \cdot|F(\beta)-F(\alpha)|+V(g ;[\alpha, \beta]) \mathcal{O}(F ;[\alpha, \beta])$ whenever $a \leq \alpha<\beta \leq b$.

(ii) $\mathcal{O}(H ; P) \leq \sup _{x \in[a, b]}|g(x)| \cdot \mathcal{O}(F ; P)+V(g ;[\alpha, \beta]) \cdot \mathcal{O}(F ;[\alpha, \beta])$, whenever $P \subseteq[\alpha, \beta] \subseteq[a, b]$.

Proof. (i) $|H(\beta)-H(\alpha)|=\mid(F(\beta)-F(\alpha)) \cdot g(\beta)+(g(\beta)-g(\alpha)) \cdot F(\alpha)-$ $(\mathcal{R S}) \int_{\alpha}^{\beta} F(t) d g(t)|=|(F(\beta)-F(\alpha)) \cdot g(\beta)+(\mathcal{R S}) \int_{\alpha}^{\beta}(F(\alpha)-F(t)) d g(t) \mid \leq$ $|F(\beta)-F(\alpha)| \cdot \sup _{x \in[a, b]}|g(x)|+V(g ;[\alpha, \beta]) \cdot \mathcal{O}(F ;[\alpha, \beta])$.

(ii) This follows by the definition of the oscillation and applying (i) to each $\alpha^{\prime}, \beta^{\prime} \in P$, where $\alpha \leq \alpha^{\prime}<\beta^{\prime} \leq \beta$.

Lemma 12. Let $F, g, H:[a, b] \rightarrow \mathbb{R}$ be such that $F$ is continuous, $g \in V B$ and $H(x)=F(x) g(x)-(\mathcal{R S}) \int_{a}^{x} F(t) d g(t)$. Let $P \subseteq[a, b]$ and let $n$ be a positive integer. Theneach of the following hold.

(i) $H$ is continuous on $[a, b]$.

(ii) If $F$ is Lipschitz on $[a, b]$, then $H$ is Lipschitz on $[a, b]$.

(iii) If $F \in A C$ on $P$, then $H \in A C$ on $P$.

(iv) If $F \in A C^{*}$ on $P$, then $H \in A C^{*}$ on $P$.

(v) If $F \in A C_{n}$ on $P$, then $H \in A C_{n}$ on $P$. (This is a slight extension of Lemma 5.23.1 of [5].)

(vi) If $F \in S A C_{n}$ on $P$, then $H \in S A C_{n}$ on $P$. (This is a slight extension of Lemma 5.24.1 of [5].)

Proof. (i) This follows immediately from Lemma 11.

(ii) Let $c>0$ be a constant given by the fact that $F$ is Lipschitz on $[a, b]$. Let $[\alpha, \beta] \subseteq[a, b]$. Since $F$ is continuous on $[a, b]$, there exists $\left[\alpha_{o}, \beta_{o}\right] \subseteq[\alpha, \beta]$ such that

$$
\mathcal{O}(F ;[\alpha, \beta])=\left|F\left(\beta_{o}\right)-F\left(\alpha_{o}\right)\right| \leq c\left(\beta_{o}-\alpha_{o}\right) \leq c(\beta-\alpha) .
$$

By Lemma 11 (i) and (7), we have

$$
\begin{aligned}
& |H(\beta)-H(\alpha)| \leq \sup _{x \in[a, b]}|g(x)| \cdot c(\beta-\alpha)+V(g ;[\alpha, \beta]) \cdot c(\beta-\alpha) \\
\leq & (\beta-\alpha) \cdot c \cdot\left(\sup _{x \in[a, b]}|g(x)|+V(g ;[\alpha, \beta])\right) .
\end{aligned}
$$

Therefore $H$ is Lipschitz on $[a, b]$. 
(iii) and (iv) follow by Lemma 11 (see also Lemma 2 of [3], p. 31).

(v) Let $M=\sup _{x \in[a, b]}|g(x)|+V(g ;[\alpha, \beta])$ and let $\epsilon>0$. Since $F \in A C_{n}$ on $P$, by Proposition 2.28.1 of [5], it follows that there exists a $\delta>0$ such that if $\left\{I_{k}\right\}, k=1,2, \ldots, s$ are nonoverlapping closed intervals with each $P \cap I_{k} \neq \emptyset$, and $\sum_{k=1}^{s}\left|I_{k}\right|<\delta$, then for each $k$ there exists $\left\{P_{k j}\right\}, j=1,2, \ldots, n$ such that $P \cap I_{k}=\cup_{j=1}^{n} P_{k j}$ and $\sum_{k=1}^{s} \sum_{j=1}^{n} \mathcal{O}\left(F ; P_{k j}\right)<\epsilon /(2 M)$. Let $\eta>0$ such that $\mathcal{O}(F ; I)<\epsilon /(2 n M)=\epsilon_{1}$, whenever $I$ is a closed subinterval of $[a, b]$ with $|I|<\eta$. (This is possible because $F$ is continuous on $[a, b]$.) Let $\delta_{1}=\min \{\delta, \eta\}$. Then $\mathcal{O}\left(F ; I_{k}\right)<\epsilon_{1}$, for each $k$. By Lemma 11 (ii) it follows that $\mathcal{O}\left(H ; P_{k j}\right) \leq M \cdot \mathcal{O}\left(F ; P_{k j}\right)+V\left(g ; I_{k}\right) \cdot \mathcal{O}\left(F ; I_{k}\right)$. Hence

$$
\begin{aligned}
& \sum_{k=1}^{s} \sum_{j=1}^{n} \mathcal{O}\left(H ; P_{k j}\right) \leq M \cdot \sum_{k=1}^{s} \sum_{j=1}^{n} \mathcal{O}\left(F ; P_{k j}\right)+n \epsilon_{1} \cdot \sum_{k=1}^{s} V\left(g ; I_{k}\right) \\
& \leq M \epsilon /(2 M)+n \epsilon_{1} M<\epsilon .
\end{aligned}
$$

Therefore $H \in A C_{n}$ on $P$.

(vi) The proof is similar to that of (v) using Proposition 2.34.1 of [5] instead of Proposition 2.28.1 of [5].

Theorem 7. Let $F, g, H:[a, b] \rightarrow \mathbb{R}$ be such that $F$ is continuous, $g \in V B$ and $H(x)=F(x) g(x)-(\mathcal{R S}) \int_{a}^{x} F(t) d g(t)$. Then each of the following hold.

(i) $H$ is continuous on $[a, b]$.

(ii) If $F$ is Lipschitz on $[a, b]$, then $H$ is Lipschitz on $[a, b]$ and $H^{\prime}(x)=$ $g(x) F^{\prime}(x)$ a.e. on $[a, b]$.

(iii) If $F \in A C$ on $[a, b]$, then $H \in A C$ on $[a, b]$ and $H^{\prime}(x)=g(x) F^{\prime}(x)$ a.e. on $[a, b]$.

(iv) If $F \in A C G^{*}$ on $[a, b]$, then $H \in A C G^{*}$ on $[a, b]$ and $H^{\prime}(x)=g(x) F^{\prime}(x)$ a.e. on $[a, b]$.

(v) If $F \in A C G$ on $[a, b]$ and is derivable a.e. on $[a, b]$, then $H \in A C G$ on $[a, b]$ and $H^{\prime}(x)=g(x) F^{\prime}(x)$ a.e. on $[a, b]$.

(vi) If $F \in A C G$ on $[a, b]$, then $H \in A C G$ on $[a, b]$ and $H_{a p}^{\prime}(x)=g(x) F_{a p}^{\prime}(x)$ a.e. on $[a, b]$.

(vii) If $F \in \mathcal{F}$ on $[a, b]$, then $H \in \mathcal{F}$ on $[a, b]$ and $H_{a p}^{\prime}(x)=g(x) F_{a p}^{\prime}(x)$ a.e. on $[a, b]$.

(viii) If $F \in S \mathcal{F}$ on $[a, b]$, then $H \in S \mathcal{F}$ on $[a, b]$ and $H_{a p}^{\prime}(x)=g(x) F_{a p}^{\prime}(x)$ a.e. on $[a, b]$. 
(ix) If $F \in S A C G$ on $[a, b]$, then $H \in S A C G$ on $[a, b]$ and $H_{a p}^{\prime}(x)=g(x) F_{a p}^{\prime}(x)$ a.e. on $[a, b]$.

Proof. That $H_{a p}^{\prime}(x)=g(x) F_{a p}^{\prime}(x)$ a.e. on $[a, b]$ or $H^{\prime}(x)=g(x) F^{\prime}(x)$ a.e. on $[a, b]$ follows easily (see for example [5], Theorem 5.23.3). Now the other assertions follow by the linearity of the $\mathcal{R S}$-integral in the second argument, and by Lemma 12 .

Remark 9. Here are some special cases of $\mathcal{P}_{\text {int }}([a, b])$.

(i) $A C_{\text {int }}([a, b])=\{f:[a, b] \rightarrow \overline{\mathbb{R}}: f$ is Lebesgue integrable on $[a, b]\}$.

(ii) Lipint $([a, b])=\{f:[a, b] \rightarrow \overline{\mathbb{R}}: f$ is measurable and bounded a.e. on $[a, b]\}$.

(iii) $\left(A C^{*} G \cap \mathcal{C}\right)_{\text {int }}([a, b])=\left\{f:[a, b] \rightarrow \overline{\mathbb{R}}: f\right.$ is $\mathcal{D}^{*}$-integrable on $\left.[a, b]\right\}$.

(iv) $\left(A C G \cap \mathcal{C} \cap \Delta_{\text {a.e. }}\right)_{\text {int }}([a, b])=\{f:[a, b] \rightarrow \overline{\mathbb{R}}: f$ is Khintchine-integrable on $[a, b]\}$.

(v) $(A C G \cap \mathcal{C})_{\text {int }}([a, b])=\{f:[a, b] \rightarrow \overline{\mathbb{R}}: f$ is $\mathcal{D}$-integrable on $[a, b]\}$.

(vi) $\left(S \mathcal{F} \cap \mathcal{C} \cap \Delta_{\text {ap a.e. }}\right)_{\text {int }}([a, b])=\{f:[a, b] \rightarrow \overline{\mathbb{R}}: f$ is $S \mathcal{F}$-integrable on $[a, b]\}$. (For the definition of the $S \mathcal{F}$-integral see [5], p. 210.)

(vii) $\left(\mathcal{F} \cap \mathcal{C} \cap \Delta_{\text {ap a.e. }}\right)_{\text {int }}([a, b])=\{f:[a, b] \rightarrow \overline{\mathbb{R}}: f$ is Foran-integrable on $[a, b]\}$. (For the Foran integral $\mathcal{F}$, see $[6]$ or $[5]$, p. 207.)

(viii) $\left(S A C G \cap \mathcal{C} \cap \Delta_{\text {ap a.e. }}\right)_{\text {int }}([a, b])=\{f:[a, b] \rightarrow \overline{\mathbb{R}}: f$ is $S A C G$-integrable on $[a, b]\}$ (SACG is the Iseki sparse integral, see [8] and [9]).

\section{References}

[1] A. Alexiewicz, Linear functionals on Denjoy integrable functions, Coll. Math. 1 (1948), 289-293.

[2] A. M. Bruckner, Differentiation of real functions, Lect. Notes in Math., vol. 659, Springer-Verlag, 1978.

[3] V. G. Člidze and A. G. Džvaršeišvili, The theory of the Denjoy integral and some applications, World Scientific, 1978.

[4] N. Dunford and J. T. Schwartz, Linear operators, Interscience, 1958.

[5] V. Ene, Real functions - current topics, Lect. Notes in Math., vol. 1603, Springer-Verlag, 1995. 
[6] J. Foran, An extension of the Denjoy integral, Proc. Amer. Math. Soc. 49 (1975), 359-365.

[7] R. Henstock, Linear analysis, Butterworth London, 1968.

[8] K. Iseki, On the normal integration, Nat. Sci. Rep. Ochanomizu Univ. 37 (1986), 1-34.

[9] K. Iseki, On the sparse integration, Nat. Sci. Rep. Ochanomizu Univ. 37 (1986), 91-99.

[10] L. V. Kantorovici and G. P. Akilov, Analiză funcţională, Editura ştiinţifică şi enciclopedică, 1986, (in romanian).

[11] H. Kestelman, Modern theories of integration, 2nd ed. ed., Dover New York, 1960.

[12] P. Y. Lee, Lanzhou lectures on Henstock integration, World Scientific, Singapore, 1989.

[13] I. P. Natanson, Theory of functions of a real variable, 2nd. rev. ed., I Ungar, New York, 1961.

[14] K. Ostaszewski, Topology for the spaces of Denjoy integrable functions, Real Anal. Exchange 9 (1983-1984), no. 1, 79-85.

[15] S. Saks, Theory of the integral, 2nd. rev. ed., vol. PWN, Monografie Matematyczne, Warsaw, 1937.

[16] W. L. C. Sargent, On the integrability of a product, J. London Math. Soc. 23 (1948), 28-34. 